\title{
Evolución del concepto de seguridad en la República del Ecuador: desde una perspectiva de seguridad nacional hacia la seguridad integral
}

\author{
Ángel Giovany Lucio VásQueZ*
}

\section{RESUMEN}

El concepto de seguridad integral incluido en la Constitución de la República del Ecuador del 2008 se ha convertido en un nuevo paradigma en el marco de la conceptualización de la política pública frente a las amenazas que afectan al individuo, el entorno y la organización política ecuatoriana.Así pues, esta nueva percepción procura sustituir el antiguo concepto de seguridad nacional desarrollado durante la Guerra Fría, centrado en la protección del estado, hacia una noción más antropocéntrica que amplía la noción de seguridad a partir de la protección del individuo y su relación con el medio ambiente, en el marco de un estado garante de derechos. El estudio analiza la evolución del concepto de seguridad nacional hacia la nueva construcción de seguridad integral. En este marco se utilizará el método descriptivo que permitirá una recolección sistémica de las diferentes normativas que coadyuvarán a describir las características de cada uno de estos momentos históricos.

\section{Palabras Clave}

Seguridad Nacional; Seguridad Integral; Evolución; Guerra Fría; Globalización.

\section{Tithe}

Evolution of the security concept in the Republic of Ecuador: From National Security to Integral Security

\begin{abstract}
The concept of integral security to be found in the Constitution of the Republic of Ecuador of 2008 has become a new paradigm in the conceptualization of public policy facing threats that affect people, the environment, and Ecuadorian political organization. This new perception seeks to replace the old concept of national security developed during the Cold War, which was focused on the protection of the State, in favour of a more anthropocentric and broader notion of security, starting from the protection of the individual and its relationship with nature. This is conceived, moreover, within the framework of the State as guarantor of rights. The present study analyzes the evolution of the concept of national security towards the new construction of integral security. For this end, the descriptive method will be used, which will allow for a systemic collection of the different regulations in order to describe the characteristics of each of these historical moments.
\end{abstract}

\section{KEYWORDS}

National Security; Integral Security; Evolution; Cold War; Globalization.

* Ángel

Giovany LUCIO

VÁSQUEZ,

Licenciado en

Ciencia Politica y

Maestría en Estu-

dios Internacionales

por el Instituto de

Estudios Europeos

de la Universidad

de Paris VIII y ob-

tuvo una Maestría

en Gobernanza

Europea por Scien-

ce Po-Grenoble,

en cuanto a su

formación comple-

mentaria en temas

de seguridad ha

sido egresado del

Centro de Estudios

Hemisfericos de

Defensa en EEUU

y de la Escuela

Militar Francesa,

actualmente funge funciones como docente de Derecho Internacional y de Integración en la Facultad de

Derecho de la

Universidad Central del Ecuador.

Recibido:

24/10/2019

Aceptado:

19/12/2019

\section{DOI:}

https://doi.org//0.15366/relacionesinternacionales2020.43.009

Formato de citación recomendado: LUCIOVÁSQUEZ, Ángel Giovany, “Evolución del concepto de seguridad en la República del Ecuador: desde una perspectiva de seguridad nacional hacia la seguridad integral”, en Relaciones Internacionales, n 43, 2020, pp. I7I188 


\section{ntroducción}

Cuando la transgresión del sentimiento de seguridad ocurre se aprecia y se reflexiona sobre su valía. En este marco, un caso ejemplificador que revela bien este postulado tuvo lugar en el momento en que el Presidente de los Estados Unidos, Franklin D. Roosevelt se dirigió de manera escrita al Congreso y efectuó una alocución sobre el Estado de la Unión' en el año 1944. En el texto, denominado Segunda Carta de los Derechos Humanos, en un fragmento arguye sobre los factores adversos que llevaron a que se desencadene el social nacionalismo alemán y las consecuencias nefastas a la seguridad internacional: "Hemos llegado, sin embargo, a una comprensión más clara de que la verdadera libertad individual no puede existir sin seguridad e independencia económica. Los hombres necesitados no son libres. Las personas que tienen hambre, las personas que no tienen empleo son la materia prima de la que están hechas las dictaduras".

Efectivamente, la interpretación del concepto de seguridad en este caso específico se comprende a partir del análisis del contexto socio histórico. Así pues, este enfoque efectúa un análisis del periodo d' avant guerre, donde la situación económica resultado de los Tratados de Versalles pusieron de rodillas al Imperio Alemán ${ }^{2}$ —antes los países de la Entente—, lo cual creó un sentimiento de inseguridad colectivo entre los ciudadanos de este país y permitió la llegada de Hitler al poder, basándose en el despertar de un nacionalismo alemán que vio en las medidas económicas draconianas y en la crisis mundial de los años 20 el sustento para una contrarréplica nacionalista a partir de la industrialización y expansión militar en Europa.

En este marco, una vez concluida la Segunda Guerra Mundial, los aliados tenían en mente la búsqueda de un nuevo paradigma donde el uso de la fuerza armada sería prohibida ${ }^{3}$ y que la guerra como amenaza no se aplicaría mas como instrumento de las Relaciones Internacionales (RRII) ${ }^{4}$. En consecuencia, la visión del autor del discurso precedente, establecía que la seguridad estaría ligada a la libertad individual en el orden material y, por tanto, a la adquisición de oportunidades para el disfrute de bienes para una futura reconstrucción de Europa y el desarrollo de ciertas regiones estadounidenses. De esta forma, el valor denominado libertad de orden económico va íntimamente ligado al concepto de seguridad. En este sentido, el exmandatario estadounidense hace mención de que un estado de bienestar necesariamente necesita un ente político que obstruya cualquier amenaza sobre los valores individuales y sociales, los cuales se encontrarían circunscriptos en el ámbito ideológico. En este punto, dos variables se combinan para minimizar las amenazas en torno a la seguridad. Por un lado, el desarrollo de un estado de bienestar de vertiente capi-

\footnotetext{
Libres de temor y libres de necesidad (Fredom from fear and fredom from want), en estas palabras Rossevelt presenta su Segunda Carta de Derechos. El texto completo se encuentra en http://www.fdrlibrary.marist.edu/archives/address_text.html

2 John Meynard Keynes, delegado del gobierno inglés a la suscripción del Tratado de Versalles explicó su postura contraria a esta decisión de sanciones desproporcionadas e inequitativas a partir de la publicación de su libro denominado "las consecuencias económicas de la paz".

3 Es evidente que la distribución del poder en el nuevo contexto geopolítico establecía que hacer la guerra sería una potestad del Consejo de Seguridad, es decir de los cinco ganadores de la Segunda Guerra Mundial, y que ningún otro estado podría disponer de este privilegio sopena de una sanción.

4 En La Carta del Atlántico del 14 de Agosto de 194I se esboza, por parte de Roosevelt, los principios de un nuevo orden internacional, enseguida, el I de enero de 1942 en Washington se reúnen una veintena de lideres mundiales y establecen la Declaración de las Naciones Unidas. Posterior a esto, en la Conferencia de Moscú en 1943, EE.UU., Reino Unido, URSS y China proclaman la necesidad de establecer una organización general fundada sobre la premisa de igualdad soberana. En consecuencia, en la conferencia de Teherán, Churchill, Roosevelt y Stalin convienen en crear la organización internacional que se hará realidad de manera técnica en la conferencia de Dumbarton Oaks de 1944. Por último, en la Conferencia de Yalta de 1945, se resuelven cuestiones como la representación de la URSS por tres estados y convienen realizar una conferencia constitutiva en San Francisco en abril-junio de 1945.Véase: Maurice, Les relations internationales depuis 1945, Armand Colin, París, 1996, p. 8
} 
talista y, por otro lado, la materialización de derechos económicos a partir de oportunidades de desarrollo individual y colectivo. Evidentemente, la protección de estas se efectuaría por parte del leviatán estatal. Es importante subrayar estos valores puesto que traducirá las amenazas de otro tipo de valores como la igualdad que profetizaba el comunismo.

Subsecuentemente, la división del mundo a partir del inicio de la Guerra Fría entre 19451955 establece una lucha por el control de territorios por parte de las ideologías dominantes durante la segunda mitad del siglo XX. Esta situación estableció doctrinas de pensamiento disímiles, por un lado, la visión occidental que proponía la premisa de libertad individual a través de un sistema donde la propiedad privada primaba, y por otro lado, la postura soviética que promulgaba la igualdad económica a partir de una economía planificada. En este marco, cada potencia trató de influenciar ideológicamente en cada región con el fin de mantenerla bajo su tutela. Es así como América Latina fue el escenario de una confrontación ideológica sobre todo en América Central y el Cono Sur en la medida que cada grupo buscaba grupo tomar las riendas del dominio político de cada país. Para el efecto, Estados Unidos estableció una doctrina enmarcada en sus intereses nacionales que se expandió a su zona de influencia; en un primer momento, en Europa y Asia, para replicarla en su Mediterráneo próximo ${ }^{5}$, y posteriormente, América del Sur, la cual se denominó doctrina de la seguridad nacional.

Así pues, el presente estudio analiza la evolución del concepto de seguridad nacional que se concibió en los años cincuenta en Estados Unidos y se expandió durante los años setenta a la República del Ecuador; hasta el concepto de seguridad integral que fue adoptado en la Constitución del 2008. En efecto, el análisis determina las diferencias entre las amenazas entre los espacios-tiempo, así como realiza un comparativo sobre los sujetos que pesaba las amenazas y los valores en confrontación.

\section{La desconstrucción del concepto de seguridad nacional}

El autor francés Fernand Braudel se cuestiona sobre el estudio de la historia, así pues, se pregunta: ¿Cómo organizar un cúmulo de hechos y de explicaciones? Si habría que escoger, yo escogería los equilibrios y desequilibrios profundos a largo plazo, señalaba el autor de la Dinámica del Capitalismo. De esta manera, las realidades sociales son comprendidas a partir del estudio de la historia en tiempo largos, puesto que permiten reconocer las estructuras de poder que se constituyen. En esta línea, el presente estudio explica la transformación del concepto de seguridad en el marco de dos cambios estructurales e históricos en la esfera internacional. En primer lugar, la Guerra Fría que va desde 1945 hasta 1991. En segundo lugar, a partir del proceso de tercera globalización, que inició desde los años noventa hasta la actualidad; esto con el fin de verificar cómo los cambios de ideologías reformulan el concepto de seguridad.

En este marco, para iniciar, es preciso desconstruir ${ }^{6}$ el concepto de seguridad o dicho de

\footnotetext{
5 Entre dos tierras, el mediterráneo de Estados Unidos como zona de influencia se encuentra en la zona geográfica entre América Central y las Antillas sobre todo Cuba y Puerto Rico.

6 Jacques Derrida establece que lo imposible es aquello que llega sin premeditar, por tanto, es en un momento de crisis que se desconstruye o desestabiliza lo que se considera natural. En este sentido, uno de los elementos de la deconstrucción es no naturalizar lo que se cree que es natural. Es decir, no asumir que lo que está condicionado por la historia, por las instituciones, o la sociedad es natural. Por ejemplo, el concepto de soberanía nacional absoluta puede ser deconstruida en su concepción indivisible a partir de una soberanía compartida de la Unión Europea. Por tanto, el concepto de seguridad puede ser desconstruido y este análisis mostrará la crisis del concepto establecido durante la Guerra Fría hacia un concepto más ligado al proceso de globalización.
} 
otro modo, preguntarse qué se entiende realmente por el concepto de seguridad o cómo y quién construye el concepto como tal. Cabe señalar que la referida noción se ha usado excesivamente para definir diferentes realidades de la vida cotidiana; para complicar aún mas su sentido multiforme, se han agregado adjetivos para categorizar los sectores de actividad afectados por amenazas heterogéneas. De esta manera, términos como seguridad interna, integral, humana, nacional, social, colectiva, muestran una amalgama de concepciones para definir realidades complejas. Sin embargo, al final del día, estas diferentes concepciones presentan los valores que resguarda una sociedad ante las amenazas que se desarrollan en cada unidad en las relaciones sociales, es decir: individuos, sociedades y estados en el ámbito internacional ${ }^{7}$. Cabe también cuestionar la idea de saber quién determina qué es lo que se convierte en amenaza, o más precisamente, quién determina los valores que hay que proteger como nación. Estas concepciones son evolutivas pues, ‘acaso es posible cambiar en momentos de crisis los valores en juego y reconstruir los elementos de un nuevo concepto?

De esta manera, el deseo de seguridad de los hombres frente a los peligros que representan la naturaleza, sus semejantes, los estados y últimamente la tecnología, han sido una base fundamental en la formación de entidades políticas ${ }^{8}$.Así, tomando en cuenta la dificultad de delimitar los intereses, para Arnold Wolfers el concepto de seguridad se define como un símbolo ambiguo", puesto que depende del interés nacional subjetivo y especulativo que influencia en la política extranjera. Por otra parte, Gallie ${ }^{10}$ lo describe como un concepto esencialmente disputado por la ideología que puede estar detrás de él. Finalmente, Khatchadourian estima que esta noción podría ser considerada como un concepto vago ", ya que ofrece varias definiciones sin que ninguna establezca su real significado.

En efecto, el concepto de seguridad y el significado del apelativo nacional es difícil de discernir por la dificultad en determinar qué tipo de intereses se encuentran detrás, quiénes participan en su construcción y, sobre todo, cómo influencia en la política exterior. De esta manera, es imposible analizar este concepto desde una sola óptica y resulta necesario observar desde varios prismas la constitución de esta figura.

Por su parte, con un análisis más preciso, Wolfers estima que por un lado existe "la parte objetiva que mide la ausencia de amenazas que pesan sobre los valores adquiridos y, por otro lado, la parte subjetiva que indica la ausencia de temor de que estos valores sean atacados”|2. De

\footnotetext{
Para Barry Buzan existen tres niveles de análisis además del estado céntrico como lo establecen los teóricos realistas. Así pues, determina que los individuos, los estados y el sistema son los niveles de análisis a tomar en cuenta.Además, el concepto de seguridad provee una síntesis que reconcilia las escuelas de poder y paz. BUZAN, Barry, "Peace, Power, and Security: Contending Concepts in the Study of International Relations" en Journal of Peace Research, vol. 21 , n² 2, 1984, pp. I09-25.

8 En este sentido, la tradición filosófica presentada al estado como garante de la seguridad nacional nació en el siglo XVII a través de autores del contrato social como Hobbes, Rousseau, Montesquieu. Esta visión propone que una entidad política pueda regular la violencia legítima y enfrentar las amenazas de la época, por un lado, estas se reflejan en la relación de conflicto entre una burguesía naciente versus la nobleza en decadencia y otro por la aparición de un nuevo proletariado resultante de las migraciones rurales. En este sentido, el estado sería responsable de manejar la violencia que surge como resultado del paso del antiguo sistema económico feudal al nuevo sistema capitalista mercantilista. Este proceso se afirmará cuando la burguesía encuentre que su lugar predominante se impone como la ideología dominante a través del liberalismo político y económico en el siglo XVIII y su expansión en el siglo XIX (imperialismo).

9 WOLFERS, Arnold, “'National Security’ as an Ambiguous Symbol” en Political Science Quarterly, vol. 67, n 4, I952, pp. 48I-502.

10 GALLIE, Walter B., “Essentially Contested Concepts” en Proceedings of the Aristotelian Society, vol. 56, n I, I955, pp. I67- I98.

" KHATCHADOURIAN, Haig, "Vagueness” en The Philosophical Quarterly, vol. I2, n 47, I962, pp. I38-I 52.

12 WOLFERS, Arnold, “'National Security' as an Ambiguous Symbol” en Political Science Quarterly, vol. 67, n 4, I 952, pp. 48I-502.
} 
esta manera, en esta primera parte se analizará la parte objetiva para definir qué tipo de amenazas estuvieron en juego en la construcción de la doctrina de la seguridad nacional por parte de Estados Unidos y la posterior influencia de corrientes de pensamiento del centro mundo en las doctrinas nacionales de la periferia como la ecuatoriana. Por último, en un segundo momento se analizará la normativa de los años setenta en torno del concepto de seguridad nacional en la República del Ecuador, sus actores y su ámbito de aplicación, para determinar su relación con el concepto de seguridad nacional estadounidense.

\section{I.I. Guerra Fría y doctrina estadounidense de seguridad nacional}

En esta primera parte, el análisis se centrará en estudiar la influencia que tuvo la teoría realista de RRII en la concepción del concepto de seguridad nacional en Estados Unidos durante la Guerra Fría, con el fin de explicar la influencia que tuvo esta corriente de pensamiento en la concepción de seguridad nacional en la República del Ecuador.

La seguridad, siendo un término de uso corriente, sería obvio pensar que no se tendría que fundamentar acerca de su definición, puesto que dispone de un sentido general en su uso. Sin embargo, esta realidad se refuta porque el significado que se otorgue a este concepto, como se ha explicado, depende de las creencias culturales y el marco histórico en que estas se desarrollen. Es decir, los valores individuales o colectivos que se convierten en intereses según la concepción de las élites dominantes, los cuales prevalecen y crean una ideología absoluta de pensamiento.

Así pues, durante el período de la Guerra Fría, la seguridad para el Occidente ${ }^{13}$ se concebía a partir de intereses nacionales como la salvaguardia de la libertad política traducida en la protección del sistema democrático y, por otro lado, la libertad económica a través del respeto de la propiedad privada y el libre emprendimiento; valores que eran ungidos por el sistema capitalista a partir de la materialización de derechos civiles, políticos y económicos al interior, y a la imposición a través de la fuerza a nivel internacional ${ }^{14}$ de un mesianismo estadounidense.

En consecuencia, surgen teorías ${ }^{15}$ que intentan explicar la influencia y efectos de la expansión del comunismo como amenaza. La principal teoría, denominada realista, fue muy apreciada en la época y determina que la seguridad, el poder, la anarquía y el equilibrio de poderes son conceptos claves en el análisis de las relaciones en la esfera internacional, sobre todo en un marco tan álgido de confrontación bipolar interpuesta. En efecto, la seguridad se traduce como la supervivencia del estado-nación y surge como el interés principal de individuos y grupos específicos, sin percibirse como una moralidad de orden universal ${ }^{16}$. Por tanto, la seguridad es la búsqueda de

13 Cabe señalar que el Pacto de Varsovia también establecía su propia concepción de seguridad nacional oficial con una doctrina MarxistaLeninista que veía una antinomia entre sistemas sociopolíticos y económicos idénticos y diferentes. Para una mejor comprensión ver: RUBIN F."The Theory and Concept of National Security in the Warsaw Pact Countries" en International Affairs, vol. 58, n 4, 1982 , pp. 648-657.

14 Cabe argumentar que la fuerza militar como medio para adherirse a los valores de cada gran potencia mundial en el marco de la bipolaridad fue utilizado tanto por la Unión Soviética como por los Estados Unidos.

${ }^{15}$ Es preciso señalar que no solo la teoría realista y neorrealista explica el concepto de seguridad. Teorías como la idealista expresa que la seguridad es una construcción que se concretiza a partir de la cooperación entre estados. Por otro lado, el constructivismo establece que es el resultado de las interacciones de varios actores, con valores sociales e identidades dan forma a estas relaciones.

${ }^{16}$ E. H. Carr, en su obra The Twenty Years Crisis efectúa una critica a los teoristas idealistas y señala que la moralidad es relativa y no universal, porque esta se encuentra compuesta por intereses y sirve para justificar y mantener la posición dominante. Así pues, la moralidad en el ámbito internacional se traduce en la creación de normas que se imponen por parte de los grupos dominantes de naciones hacia otras naciones por medio de la coerción. Véase: CARR, Edward H., The Twenty Years' Crisis, 1919-1939: An Introduction to the Study of International Relations, Macmillan and Co., Limited, Londres, 1940. 
poder e influencia en un mundo anárquico ${ }^{17}$, sin autoridad superior que regule las interacciones estatales. De esta manera, la expansión económica, cultural y cuasi territorial ${ }^{18}$, se materializa por medio de la guerra con el fin de contener a su oponente. En este sentido, Hans Morgenthau señala que la consecución de poder y riqueza permite reducir la vulnerabilidad a partir del equilibrio de poder ${ }^{19}$ entre potencias; esto en gran parte debido a la multiplicidad y antagonismo entre estados. Por consiguiente, la respuesta a la directa oposición y la competición es establecer métodos que equilibren el peso de cada potencia en las RRII. Es así que dividir y regular, establecer compensaciones, disponer de armamentos, realizar alianzas, son elementos que conllevan a la conformación de sistemas dominantes y dependientemente equilibrados ${ }^{20}$. En cambio, para Stephen Walt, el estado es la unidad principal de análisis y su principal objetivo es la integridad territorial y la seguridad física de sus habitantes ${ }^{21}$; es decir, evitar ataques armados o una posible coerción por parte de otro estado. Por tanto, la seguridad para los realistas estaba íntimamente ligada a la capacidad de resguardar y expandir los valores mediante la guerra. Así, para Raymond Aron, la paz sería solamente una suspensión más o menos durable de modalidades violentas de rivalidad entre unidades políticas ${ }^{22}$.

En este sentido, la base teórica en que se construye el concepto de seguridad nacional estadounidense en su concepción teórica está ligado a una relación de búsqueda de poder para la supervivencia de los estados a través del uso de la fuerza armada. Esto es así sobre todo si se hace énfasis en un periodo de confrontación entre dos grandes superpotencias. En este marco, para Bertkowitz \& Bock, la seguridad nacional se establece como "la capacidad que dispone un país a través de sus instituciones políticas para proteger los valores internos contra las amenazas externas" ${ }^{23}$. Sin embargo, para Frank N. Trager el propósito es más extenso, puesto que no solo protege sino expande los valores de cada nación; es decir, lo presenta como un concepto clave en el desarrollo del imperialismo estadounidense. En este sentido, el autor presenta a la seguridad nacional como parte de la política gubernamental que tiene como "objetivo central la creación de condiciones nacionales e internacionales favorables para la protección y extensión de los valores vitales nacionales contra los adversarios existentes o potenciales ${ }^{24 ”}$. En este ámbito de acción, para Wolfers, la seguridad nacional no es solo la protección de valores previamente adquiridos sino las expectativas sobre el futuro y el resultado de los valores que serán experiencias posteriores. Por tanto, la seguridad no solo evita la pérdida, sino también la prevención. En este marco,

17 Para Kenneth Waltz, un autor neorrealista, la anarquía es un concepto clave que es el resultado de la inseguridad y ganancias desiguales de los estados. En un sistema de autoayuda, las consideraciones de seguridad subordinan la ganancia económica al interés político. Por tanto, en la era nuclear bipolar internacional basado en dos superpotencias, Estados Unidos y la Unión Soviética no era estable sino mas bien persistiría. En:WALTZ, Kenneth N., Man, the State and War:A Theoretical Analysis, Columbia University Press, Nueva York, 1959.

${ }^{18}$ En efecto, la guerra por medio de terceros llevó a los EE.UU. y a la URSS a una confrontación en varios campos de batalla, sobre todo para los norteamericanos este fenómeno no significó una expansión de territorio sino una influencia ligada a la subordinación con las élites locales y la implementación de un sinnúmero de bases militares en territorios semisoberanos. En este marco consultar el artículo de GOLUB, Philip, "Imperial politics, imperial will and the crisis of US hegemony" en Review of International Political Economy, vol. II, n 4, 2004, pp. 763-786.

19 El término "equilibrio de poder" se utiliza en el texto con cuatro significados diferentes: (I) como una política dirigida a cierto estado de cosas; (2) como una lista real de asuntos; (3) como una distribución aproximadamente igual de poder; (4) como cualquier distribución de poder. MORGENTHAU, Hans J., THOMPSON, Kenneth W., y CLINTON, W. David, Politics Among Nations: The Struggle for Power and Peace, McGraw-Hill Higher Education, Boston, 2006.

20 Ibídem.

21 WALT, Stephen M., The Renaissance of Security Studies, Sage, Beverly Hills, I99I, p. 2 II.

22 ARON, Raymond, Paix et guerre entre nations, Calman Levy, París, I984, p. 158.

23 BERKOWITZ, Morton, BOCK, P. G., American National Security:A Reader in Theory and Policy, Free Press, Nueva York, 1965.

24 TRAGER, Frank N., y KRONENBERG, Philip S., National Security and American Society; Theory, Process, and Policy, National Security Education Program, University Press of Kansas, Kansas City, 1973. 
para los realistas la obtención del poder es el medio para la consecución de la seguridad nacional a partir del uso de la disuasión por la fuerza armada o lo que se denomina también defensa nacional. Esta política pública se efectiviza a partir de las atribuciones establecidas a las fuerzas armadas. Sin embargo, para una adecuada política de seguridad nacional es necesario disponer de una amplia protección de valores previamente adquiridos por medio del orden militar y no militar, en el marco interno o internacional, utilizando formas apropiadas de poder nacional. En este marco, si bien la fuerza militar es un mecanismo de poder, es necesario añadir otros elementos que podrían contribuir, como la diplomacia, la coerción económica o cultural.

Es ese sentido, cabe señalar que establecer una definición definitiva del concepto de seguridad nacional es una tarea álgida, puesto que como se ha descrito depende del contexto histórico y por ende de los valores de ese momento ${ }^{25}$, Sin embargo, como conclusión es menester indicar que todas las posiciones referidas anteriormente demuestran una escuela de pensamiento que atesoraba la protección de la nación a través del estado como ente político representativo o mas bien dicho de una elite que disponía de valores fundamentales para su conservación a través de la fuerza de coerción ${ }^{26}$ tanto en el plano nacional como internacional.

\section{I.2. La seguridad nacional: Competición, conflicto y respuesta militar}

En la práctica, las teorías sirven para describir la realidad de interacciones sociales, además, los teóricos intentan influenciar a los hacedores de la política pública actuando como consejeros del príncipe. Esta aseveración es muy real en la esfera pública estadounidense. Así, en la práctica, la primera iniciativa que incluye una visión realista en la política pública corresponde a la denominada doctrina de la seguridad nacional que se establece en el año de 1947 con la National Security $\mathrm{Act}^{27}$. Esta se constituye como una herramienta fundamental de la Doctrina Truman para hacer frente al comunismo que se desarrollaba especialmente en Europa, en un primer momento en el caso de Grecia y Turquía. Por lo tanto, la política exterior acentuó la idea de crear mecanismos económicos como el Fondo Marshall y, desde el plano militar, establecer alianzas como la OTAN. Otro hecho que reforzó la idea de que la seguridad nacional necesitaba el uso de la fuerza militar y de disuasión nuclear fue la primera detonación por parte de la Unión de Repúblicas Socialistas Soviéticas (URSS) de una bomba atómica en 1949. Esto estableció la política de Nuclear arms race, la cual duró cerca de medio siglo e incrementó el arsenal nuclear de las dos superpotencias de manera exponencial.

Por otro lado, otro factor que impulsó la aplicación de los fundamentos de la teoría realista fue las primeras confrontaciones en el terreno de combate después de Europa. De esta forma, la Guerra de Corea fue una pieza clave para que EE.UU. considerara que el continente asiático podría caer bajo la tutela comunista. En este marco se crea un proceso político y militar de con-

${ }^{25}$ Charles F. Herman en su artículo "Defining national security” establece cinco aspectos en el escenario de la seguridad nacional que deben ser tomados en cuenta para su protección: resultado de valores preferidos, ambiente internacional, ambiente nacional, naturaleza de las amenazas, estrategias para la aversión de amenazas.

${ }^{26}$ David Jablonsky en su artículo "The State of National Security State" establece que existen tres tipos de intereses por parte de Estados Unidos: seguridad fisica contra peligro foráneo, promoción de valores y prosperidad económica, el autor añade un cuarto que denomina el orden mundial.

27 Efectúa una reorganización de la política exterior y de la estructura militar creando instituciones como el Consejo de Seguridad Nacional, la Agencia Central de Inteligencia, el Secretariado a la Defensa y el Departamento de la Fuerza Aérea unificando las fuerzas armadas. Para una mejor comprensión revizar STUART, Douglas T., Creating the National Security State:A History of the Law That Transformed America, Princenton University Press, Princeton, 2008. 
tención ${ }^{28}$, tanto en el Océano Índico, Corea, el Sudeste Asiático y el Medio Oriente. En este primer periodo, América Latina no fue considerada como una zona de prioridad estratégica, puesto que se creía que las interrelaciones de subordinación financiera y económica que se crearon a partir de la Doctrina Monroe en el periodo de las independencias, la estrategia de Alfred T. Mahan (canal ístmico y rutas marítimas del Caribe), y cierto intervencionismo (independencia de Cuba, Puerto Rico), habían sido suficientes para controlar las élites de la región y llegar a crear nexos hasta mediados del siglo XX. Estos nexos mantuvieron de lado a otras potencias a partir de la política de buen vecino (1933-1945) creada por Roosevelt, y establecieron un aparato de cooperación interamericano concebido en los años cincuenta y sesenta, a partir del Acta de Chapultepec (1945), el Tratado Interamericano de Asistencia Reciproca (TIAR 1947) y, posteriormente, la creación de la Organización de Estados Americanos (OEA) en 1948.

Sin embargo, Eisenower 1953-1961 estimó que la inequidad y los gobiernos dictatoriales eran un caldo de cultivo para el desarrollo del comunismo en la región, por lo tanto, estableció el memorándum de Operación Panamericana y se creó el Banco Interamericano de Desarrollo. En cambio, John F. Kennedy, durante los sesenta, desarrolló una relación con América Latina centrada en una visión idealista wilsoniana de orden económico y democrático a partir de la Alianza para el Progreso y la respuesta a la intervención de la URRS en la Crisis de Misiles de Cuba, que sería la primera prueba para el neófito presidente. En este marco, no es posible comprender cómo una política exterior basada en la expansión de la democracia permitió que ciertos países como Argentina, Brasil, Perú, República Dominicana, terminaran en dictaduras militares. En efecto, el avance del comunismo tanto en Cuba como en República Dominicana, permitió a las dictaduras ser una estrategia de contención a partir de varias técnicas. De esta manera, el General Maxwell Taylor estableció un mecanismo denominado contrainsurgencia; es decir, la utilización de guerras relámpago como una contra ofensiva al comunismo utilizando a las propias fuerzas insurgentes de cada país con apoyo de la CIA, el FBI, policías, militares, y la agencia de cooperación internacional, que será la política pública utilizada por Kennedy.

Posterior al asesinato de Kennedy, el vicepresidente Lyndon Johnson, en 1969, dejó en ruinas la política estadounidense en América Latina, la cual inició con la operación Power Pack planificada para la invasión de la República Dominicana, evitando así la toma del poder del líder Juan Bosch, a quien se lo relacionaba con el comunismo. Este acto llevó a que los países de América Latina establecieran una falta de apego a las políticas expansionistas de Estados Unidos ${ }^{29}$. Posteriormente, la política de Johnson se centró sobre todo en Vietnam, Laos y Camboya. Así mismo, Nixon y Kissinger se focalizaron mayormente en Vietnam, aunque con la misma perspectiva de intervenciones directas y cortas en América Central que se tradujo en la intervención en Nicaragua sandinista. En estos periodos estudiados, Estados Unidos buscó restablecer una relación de subordinación a partir del respeto de la democracia y la cooperación económica, empero, por su

${ }^{28}$ Principal política de intervensionismo durante la Guerra Fría, que inició por parte del presidente Truman (I945- I953), la cual fue suguerida por el diplomático estadounidense George Kennan, presuponía que los intereses de la URSS no eran compatibles con los valores de EEUU, por tanto, habría que efectuar estrategias preventivas de orden militar. Esta teoría primero se aplicó en Europa y posteriormente en Asia, hasta el periodo de detente que inicia con Nixon y Henry Kissinger,y continúa con Jimmy Carter y la Guerra deVietnam. Sin embargo, después de la invasión de Afganistán, en 1979, se retoma estrategias de contención que continuarían con el Presidente Ronald Reagan que promocionó acciones de rollback en países como Nicaragua y Afganistán.

29 La presentación de los hechos históricos, teorías y doctrinas en este capítulo sobre la política exterior estadounidense han sido recopiladas del texto:TULCHIN, Joseph, “Los Estados Unidos y América Latina en la década del 60” en Estudios Internacionales, vol. 21 , n 84, I988, pp. 462-497. 
temor al comunismo, terminó apoyando a los gobiernos militares y resquebrajando la esperanza de gobiernos constituidos democráticamente, situación que ha dado como resultado la construcción de una deteriorada imagen ligada al intervencionismo estadounidense. No obstante, en su deseo de colaboración, también creó organismos internacionales de cooperación y mecanismos de transferencia de doctrinas estratégicas que fueron tomadas por los gobiernos militares.

Tabla I.- Estructura protegida por Estados Unidos durante la Guerra Fría.

\begin{tabular}{|l|l|l|l|l|}
\hline Ideología & Valores & $\begin{array}{l}\text { Tipo de estructura } \\
\text { política. }\end{array}$ & $\begin{array}{l}\text { Teoría que per- } \\
\text { mitían esta protec- } \\
\text { ción }\end{array}$ & $\begin{array}{l}\text { Normativa que regulaba } \\
\text { esta ideología. }\end{array}$ \\
\hline Capitalismo & $\begin{array}{l}\text { libertad política, } \\
\text { económica, soci- } \\
\text { al y cultural, }\end{array}$ & $\begin{array}{l}\text { Estado: democrático } \\
\text { Derecho: Protección } \\
\text { de la propiedad privada }\end{array}$ & $\begin{array}{l}\text { Teoría Realista: unidad } \\
\text { de estudio estado, po- } \\
\text { der, seguridad, anarquía }\end{array}$ & $\begin{array}{l}\text { Seguridad Nacional: Protec- } \\
\text { ción de estado, sobreaña, ac- } \\
\text { ción militar, coerción, expan- } \\
\text { sión. }\end{array}$ \\
\hline
\end{tabular}

\section{I.3. La Seguridad Nacional ecuatoriana:Transposición de intereses externos}

En este marco de Guerra Fría es natural pensar que los países de América Latina se verían influenciados por las doctrinas emanadas del centro-mundo occidental. De esta manera, la materialización del concepto de seguridad se tradujo en la adopción de un marco jurídico. En efecto, en 1979 se realiza la última transposición de la doctrina de la seguridad nacional en norma legal. El interés de la aprobación de la Ley de Seguridad Nacional en el ámbito interno era el combate a la insurgencia, la cual había dado sus primeros pasos en 1965, a partir de la creación del Movimiento de Izquierda Revolucionaria (MIR) en varios países de la región andina. Este movimiento estaría muy presente en la actividad insurgente y política en el periodo de la dictadura militar del Contralmirante Ramón Castro jijón, del Presidente interino Clemente Yerovi, del Presidente Otto Arosemena Gómez y, sobre todo, en el Quinto Velasquismo, donde planificaron manifestaciones de orden estudiantil, secuestros de personas, de aviones y asaltos a instituciones financieras. Por otro lado, existía ya una cooperación con otros grupos subversivos regionales como Montoneros de Argentina,Túpac Amaru de Uruguay, el M-19 de Colombia, entre otros.Además, nuevos grupos se integrarían a la lucha por el derrocamiento de la dictadura de Velasco lbarra iniciada en 1970 , como el Partido Socialista Revolucionario Ecuatoriano y el Frente Revolucionario de Izquierda Universitaria (FRIU), lo que llevó al cierre, por parte de la dictadura velasquista, de la Universidad Central del Ecuador a través de la "ley mordaza" en I97I, y posteriormente, al derrocamiento de Velasco lbarra por parte de Rodríguez Lara.

En este marco de gran agitación social e influencia en la política nacional por parte de grupos subversivos y estudiantiles, se estableció la codificación de varios cuerpos normativos en la definitiva Ley de Seguridad Nacional en 1979, justamente en la culminación del ejercicio del poder por parte del triunvirato militar. Otro punto importante a tomar en cuenta para la adopción de la referida ley consistió en disponer de todos los recursos necesarios para hacer frente a las amenazas externas — tanto la influencia de Cuba, como la de Nicaragua- En este ámbito de la acción internacional, la conservación territorial del estado era importante. Evidentemente, la República del Ecuador había sufrido pérdidas territoriales desde su independencia a favor de Colombia, Brasil y Perú. Este último país, después de la suscripción del Tratado de Río de 194I 
-donde Ecuador perdió la mitad de su territorio en la Amazonía y su acceso directo al rio Amazonas-, continuó con las amenazas de anexión de territorio ecuatoriano, tanto en la Guerra de Paquisha de 1981, como en la del Cenepa de 1996.Así pues, la estructura de la normativa refleja la inquietud de un conflicto armado futuro a su adopción. Así mismo, en el plano internacional, la postura de una cooperación interamericana a través del TIAR valida el uso de la fuerza armada con otros países una vez un estado americano sea agredido por una potencia exterior (solidaridad con Argentina y rechazo de la intervención inglesa en las Islas de las Malvinas).

En efecto, la codificación de la Ley de Seguridad Nacional fue expedida por la Secretaria General del Consejo de Seguridad Nacional (COSENA) el 20 de julio del 1979, siendo responsabilidad del estado garantizar la supervivencia, la defensa del patrimonio y los objetivos nacionales. La normativa disponía la salvaguardia de la soberanía e integridad territorial, contrarrestando amenazas internas o externas a partir de medios económicos, políticos o militares. Cabe mencionar que durante el periodo en que se adopta la ley se ejerció un cambio radical en la política ecuatoriana, puesto que el triunvirato militar, con fecha del II de octubre de 1981, cedería el poder al Presidente Jaime Roldos Aguilera. En este caso, la ley había previsto que el presidente cumpliese un rol preponderante en la dirección de la política pública en este tema, pero sin que los militares perdieran el control en el asesoramiento a través del Consejo de Seguridad Nacional y el Comando Conjunto de las Fuerzas Armadas, a partir de las compras de armamento y formación castrense, ya que se encontraban a cargo de la ejecución y planificación de la defensa nacional.

Tabla II.- Normas para la codificación de la Ley de 1979

\begin{tabular}{|l|l|l|l|}
\hline Año & Ley & Gobierno & Presidente \\
\hline 1961 & Defensa Nacional & Civil & Velasco lbarra \\
\hline 1964 & Seguridad Nacional & Militar & Junta Militar \\
\hline 1974 & Codificación & Militar & \\
\hline 1976 & $\begin{array}{l}\text { Ley de Seguridad } \\
\text { Nacional }\end{array}$ & Militar & Junta Militar \\
\hline 1979 & $\begin{array}{l}\text { Codificación a la Ley de } \\
\text { Seguridad Nacional }\end{array}$ & Militar & Junta Militar \\
\hline $1965(3) 1972(2) 1973,1974$ & Decretos Ejecutivos & Varios & Varios \\
\hline
\end{tabular}

En este marco, en las líneas siguientes se efectuará un análisis detallado de la Ley de Seguridad Nacional. De esta manera, según esta normativa, el Presidente de la República direccionaba la política de seguridad nacional y era asistido por los Organismos Superiores de Seguridad Nacional, los cuales están constituidos por el COSENA, que formulaba y supervisaba la política de seguridad nacional. Este consejo, a su vez, está apoyado por una Secretaria General que materializa las decisiones del COSENA. Por otra parte, en este último ente se crean frentes de orden externo dirigidos por la Cancillería para defender los intereses estatales en el plano internacional. El Ministerio de defensa, particularmente, busca asegurar la protección territorial por medio de una capacidad militar instalada. En este sentido, cabe señalar que la teoría realista de RRII está muy impregnada en la elaboración de la concepción de seguridad externa, puesto que 
especifica que las RRII se basan en intereses nacionales que son obtenidos por medio de recursos militares.

Por otro lado, en el frente interno, si bien está constituido por varios ministerios, existe una fuerte influencia del Ministerio de Gobierno y Policía, que tiene a cargo la cohesión del país. Es decir, establecer un marco propicio para el desarrollo propio de los valores capitalistas que coartarían cualquier indicio de penetración por parte del comunismo, para lo cual contarían con la intervención de la Policía Nacional, entidad que fue la encargada de hacer frente a los grupos subversivos durante el Quinto Velasquismo, la dictadura y el gobierno de León Febres Cordero. En el ámbito económico se siguen las mismas líneas realistas, puesto que se busca el desarrollo nacional para hacer frente a otro tipo de doctrinas de corte comunista. Aquí cabe destacar cómo influenció la visión wilsoniana de fomento al comercio y a la democracia en las sociedades latinoamericanas, lo cual fue expandido por Kennedy mediante su Alianza para el Progreso.

Por último, el frente militar, direccionado por el Ministerio de Defensa en caso de conflicto bélico con el fin de salvaguardar la soberna nacional. Además, una de las funciones tomadas muy a pecho fue la colaboración con el desarrollo nacional. Esta última parte, en tiempos de paz, ha estado muy presente en el quehacer de las Fuerzas Armadas, quienes han desarrollado una industria militar propia.

En conclusión, dos ejes marcan esta ley. En un primer momento, la salvaguarda de valores internos frente a la amenaza externa que, sobre todo, es la lucha contra el comunismo.Y, por otro lado, en el ámbito internacional, que busca la protección de la soberanía territorial en torno a las acciones que podría tomar Perú, así como una cooperación de orden interamericana guiada por acuerdos con los EE.UU.

\section{El concepto de seguridad integral como un enfoque antropocéntrico}

El concepto de seguridad humana se instauró en el seno de la Organización de las Naciones Unidas (ONU) posterior al vacío teórico que había dejado el realismo a partir de su explicación de la Guerra Fría. En efecto, la caída del Muro de Berlín y posterior desintegración de la URSS permite a organizaciones como la ONU instaurar una visión antropocéntrica del concepto de seguridad con el fin de comprender las amenazas en su totalidad a través del prisma de la globalización. En este sentido, el Programa de Desarrollo de la ONU (PNUD) buscó una alternativa centrada en el estudio de amenazas múltiples. Así, la seguridad humana se define como "el marco analítico y de planificación que respalda respuestas más integrales y preventivas de las Naciones Unidas, que atraviesa sectores, desarrolla soluciones contextualmente relevantes y adopta asociaciones para ayudar a realizar un mundo libre de miedo, escasez e indignidad"30.

En este marco, la seguridad humana va mucho mas allá de contrarrestar las amenazas a la propiedad privada en el plano individual o en el territorio en cuanto al estado, ya que se centra en la protección de amenazas en contra de la vida humana y su dignidad en los ámbitos económico, alimentario, medio ambiente y salud, y establece niveles individuales, sociales y estatales para su concretización. En este sentido, una definición más clara del concepto de seguridad humana se realizó por parte de la ONU en los siguientes términos:"el derecho a vivir en libertad y dignidad,

\footnotetext{
${ }^{30}$ Para una comprensión del concepto de seguridad humana ver: https://www.un.org/humansecurity/what-is-human-security/
} 
libres de pobreza y desesperación [...] con la misma oportunidad de disfrutar de todos sus derechos y desarrollar plenamente su potencial humano"3!.

\section{I. La seguridad nacional como parte del concepto de seguridad integral}

En la actualidad, el concepto de seguridad nacional es un concepto muy controversial en la discusión de orden político y académico ecuatoriano. Este término fue excluido del debate nacional y dio paso a una nueva representación ideológica. De hecho, un nuevo término ha sido acuñado y nombrado como "seguridad pública y estatal", que renueva la doctrina de la seguridad para adaptarse a las demandas del mundo contemporáneo, al marco constitucional actual. Así pues, el Preámbulo de la Ley de Seguridad Publica del Estado (LSPE), establece un nuevo sistema de seguridad integrado en una perspectiva civil, dinámico y adecuado para el nuevo entorno geopolítico internacional.

Cabe señalar que este nuevo término es parte de otro concepto más amplio denominado "seguridad integral". El motivo detrás de este cambio fue destacar un nuevo desafío para la seguridad pública del estado. De hecho, la seguridad nacional controlada por el orden militar se ha convertido en la actualidad en una política dirigida por el orden civil; es decir, que la fuerza militar debe estar enmarcada con el poder democráticamente elegido. Esta posición fue una de las críticas al poder reinante de las fuerzas militares que se estableció en la esfera política durante las dictaduras que afectaron la vida democrática en la década de 1970-1980, e influyeron en la política ecuatoriana en su naciente democratización (1990-2005).

En el mismo sentido, es necesario especificar que otros motivos externos han modificado paradigma de la seguridad nacional de los años setenta. De hecho, uno de los puntos propuestos por el candidato Rafael Correa fue la reconquista de la soberanía y la independencia de potencias extranjeras. En este sentido, el término seguridad nacional que se concibió en los EE.UU. fue tildado de una política de expansión de los valores de este país entre los latinoamericanos. De esta manera, esta noción, como se ha analizado, fue conceptualizada en EE.UU. e introducida por su ejército en el entrenamiento de oficiales de policía y militares latinoamericanos en la escuela de las Américas en Panamá, para aplicar la estrategia de contrainsurgencia citada anteriormente en el marco de la contención del comunismo. Esta se desarrolló, como lo señala Hernández: "en el istmo centroamericano (Nicaragua, El Salvador, Guatemala), el Caribe (Cuba) y América del Sur (Chile,Argentina, Brasil, Uruguay) con todas las derivaciones que han ocurrido en la parte régimen dictatorial durante este período"32.

Como resultado, esta representación en el imaginario de las élites políticas y académicas, ha contribuido a la idea de que durante décadas se ha forjado una condición de subordinación por parte de los latinoamericanos a las imposiciones norteamericanas; una interferencia indeseable con el buen funcionamiento de los asuntos de su soberanía. En este sentido, la aplicación del concepto de seguridad nacional en la nueva constitución fue refutada por actores políticos de izquierda.Así lo señala Burbano de Lara:"tenían el deber de crear una nueva constitución en 2007

\footnotetext{
${ }^{31} 66^{a}$ sesión de la Asamblea General de las Naciones Unidas "Follow-up to paragraph 143 on human security of the 2005 World Summit Outcome" (A/RES/66/290). 25 de octubre de 2012.

32 HERNÁNDEZ, Rafael, “Commentary:Alliances and Dis-Alliances between the United States and Latin America and the Caribbean” en Latin American Perspectives, vol. 38, n 4, 201 I, p. 133.
} 
con el objetivo de reconstruir su tierra natal"33. El concepto de seguridad nacional, por tanto, ha dado paso $^{34}$ al concepto de seguridad pública y del estado y, en general, al de seguridad integral. La última idea es una adaptación semántica a la realidad ecuatoriana del concepto de seguridad humana desarrollado para el PNUD en la década de 1990 y expandido por potencias medias como Canadá y Japón; el concepto de seguridad humana fue concebido como una respuesta a la búsqueda de nuevos enfoques que explicaran los nuevos desafíos de seguridad en el período de la globalización posterior a la Guerra Fría.

Así, la crítica a una visión realista del concepto de "equilibrio de poder" que está estructurado por las relaciones entre los grandes de este mundo, y que busca la maximización del poder mediante alianzas, es decir, lo que Hurrell denominaba fredom from fear ${ }^{35}$, ha dado paso a un enfoque institucionalizado de la seguridad colectiva fredom from dignity. Según Shinoda, promovería la realización de los derechos desde el desarrollo de oportunidades, que se expandiría en los ámbitos económico, alimentario, sanitario, medioambiental, personal, comunitario y político. Es decir, "fredom from want" a nivel nacional e internacional ${ }^{36}$. Este enfoque de la seguridad humana en las instituciones internacionales tiene una correlación significativa con el de la seguridad integral ecuatoriana, porque en la normativa ecuatoriana se los cita indistintamente ${ }^{37}$ para mencionar la misma condición de respeto y realización de los derechos humanos.

En este sentido, la seguridad nacional que, en su esencia se concibe como la protección de la nación (territorio, población, soberanía), no desapareció totalmente de la normativa ecuatoriana, pues se transformó en el concepto de seguridad pública y del estado, ya que defenderá la soberanía y la integridad territorial ${ }^{38}$. Sin embargo, una mayor preponderancia del Ejecutivo frente a las fuerzas armadas se denotó durante el periodo 2007-20 I7. Por otro lado, como se ha explicado, el concepto de seguridad nacional ha sido reemplazado por el término seguridad pública y el estado que forma parte de la seguridad integral, siendo este último una transposición del concepto de seguridad humana desarrollado en la ONU.

\subsection{Análisis exegético de la normativa actual en el campo de la seguridad.}

Con respecto al estudio de la normativa estatal, esta se realizará a través de categorías textuales formales, como las normas legales. Está claro que el primer texto a analizar será la Constitución de la República del Ecuador. De hecho, con respecto al término seguridad, la Constitución establece 91 referencias relacionadas con el concepto de seguridad que se materializa en los diferentes dominios de la actividad humana ${ }^{39}$. Por lo tanto, como se señaló anteriormente, la normativa

\footnotetext{
33 BURBANO DE LARA, Fernando “Todo por la patria. Refundación y retorno del estado en las revoluciones bolivarianas" en Íconos. Revista de Ciencias Sociales, $n^{\circ}$ 52, 2015, pp. 19-4I.

34 La Ley de Seguridad Pública y Seguridad del Estado de 2009 (LSPE) establece en su preámbulo que es necesario renovar la doctrina de la seguridad para adaptarla a las demandas del mundo contemporáneo, al marco constitucional actual, siendo necesario tener un nuevo sistema de seguridad integral en una perspectiva civil, dinámica y adecuada para el nuevo entorno geopolítico internacional.

35 HURRELL,Andrew, "Security in Latin America” en International Affairs, vol. 74, nº 3, 1988, p. 533.

36 SHINODA, Hideaki, "The concept of human security: historical and theoretical implications" en Conflict and human security: A search for new approaches of peace-building, IPSHU English Research Report Series, $n^{\circ}$ 9, 2004, pp. 5-22.

37 En la Ley de Seguridad y el Estado hay dos veces la expresión seguridad humana y dieciséis veces el término seguridad integral. Estas denominaciones tienen como objetivo principal materializar los derechos humanos en todos los dominios de la actividad social por medio de la intervención de un estado democrático.

38 LSPE, artículo 4

39 Por lo tanto, se presentan varias denominaciones como: social, vial, integral, humana, para extranjeros, niños, legal, internacional, ciberseguridad,
} 
presenta los diferentes tipos de dominios donde se aplica este término polisémico.

En el caso específico del estudio, el término seguridad se trata de manera general e indistinta, ya que es un concepto que aborda una gran cantidad de realidades. De hecho, lo que se denomina "seguridad integral" 40 "seguridad humana" 41 se aplica en un espectro muy amplio en las relaciones sociales o más bien las engloba. Pues bien, el objetivo de esta parte es demostrar cómo la seguridad nacional se readecua al nuevo concepto de seguridad pública y estatal.Y esto último se estructura como parte del concepto general de seguridad integral que es, a su vez, influenciado por el concepto de seguridad humana desarrollado por organizaciones multilaterales.

Por lo tanto, en torno a la seguridad integral, esta debe ser materializada por el estado y dirigida por el ejecutivo. De esta manera, en el Artículo 3, numeral 8, de la CRE, se establece que el deber del estado es "garantizar a sus habitantes el derecho a una cultura de paz, a la seguridad integral y a vivir en una sociedad democrática sin corrupción”.

En este sentido, las acciones de la materialización de la seguridad integral se efectúan a partir de la legitimación activa que recae en el Presidente de la República, que debe garantizar el mantenimiento de la soberanía, la independencia del estado, el orden interno y la seguridad pública, y ejercer el liderazgo político de la defensa nacional. En el orden de la legitimación pasiva, puede ser perseguido políticamente y ser destituido por la Asamblea por delitos contra la seguridad del estado ${ }^{42}$. Por otro lado, los ciudadanos deben colaborar con el mantenimiento de la paz y la seguridad, así como defender la integridad territorial del Ecuador y sus recursos naturales ${ }^{43}$. Como resultado, la protección de la seguridad corresponde tanto a nivel estatal como individual.

En la misma línea, era esencial, en la nueva normativa, cambiar la visión y también las políticas públicas en torno a la seguridad. En este sentido, el papel de las fuerzas armadas estatales —es decir, las Fuerzas Armadas y la Policía Nacional-, actualmente se entendería que protegen los derechos, libertades y garantías de los ciudadanos, fredom from dignity ${ }^{44}$, en lugar de ser entidades que luchan por los intereses de las potencias extranjeras en un mundo bipolar fredom from fear.

Como resultado, la misión básica de las fuerzas armadas es defender la soberanía y la integridad territorial ${ }^{45}$, y la policía tiene el papel de cuidar la seguridad interna o ciudadana y el orden público para para contrarrestar el delito ${ }^{46}$. Como resultado, se hace una distinción de funciones para cada fuerza armada. Por lo tanto, el ejército tendrá la responsabilidad de proporcionar seguridad externa y la policía de seguridad interna. Sin embargo, esta disposición

${ }^{40}$ La seguridad integral se menciona solo una vez en la Constitución (CRE) en su artículo 3, numeral 8,y no existe una definición. Es necesario esperar a la adopción del LSPE para determinar su significado.

41 Seguridad humana:Título VII, Régimen del Buen Vivir, Capítulo I, artículos 340 y 393, según el último artículo, la seguridad humana se define como la garantía de la coexistencia pacífica de las personas, la promoción de una cultura de paz y la prevención de formas de violencia y discriminación y la comisión de delitos y delitos. Ella es mencionada cuatro veces en CRE.

42 Constitución de la República de Ecuador (CRE), artículo 129.

43 Ibídem, art. 83, 3-4.

44 Ibíd., art. 158.

45 Ibíd.

46 Ibíd., arts. 158 y 163.
} 
constitucional fue dejada de lado por el ex presidente de la República Rafael Correa en varias ocasiones, cuando permitió que las fuerzas armadas colaboraran con la policía en la lucha contra la delincuencia común, especialmente en estados de excepción. Sin embargo, cabe señalar que en la última protesta social con el actual mandatario en el mes de octubre 2019 , el manejo de la violencia legítima del estado hacia los manifestantes por parte del ejercito fue destacado.

Cuadro I.- Normas que regulan la Seguridad Pública y del Estado.

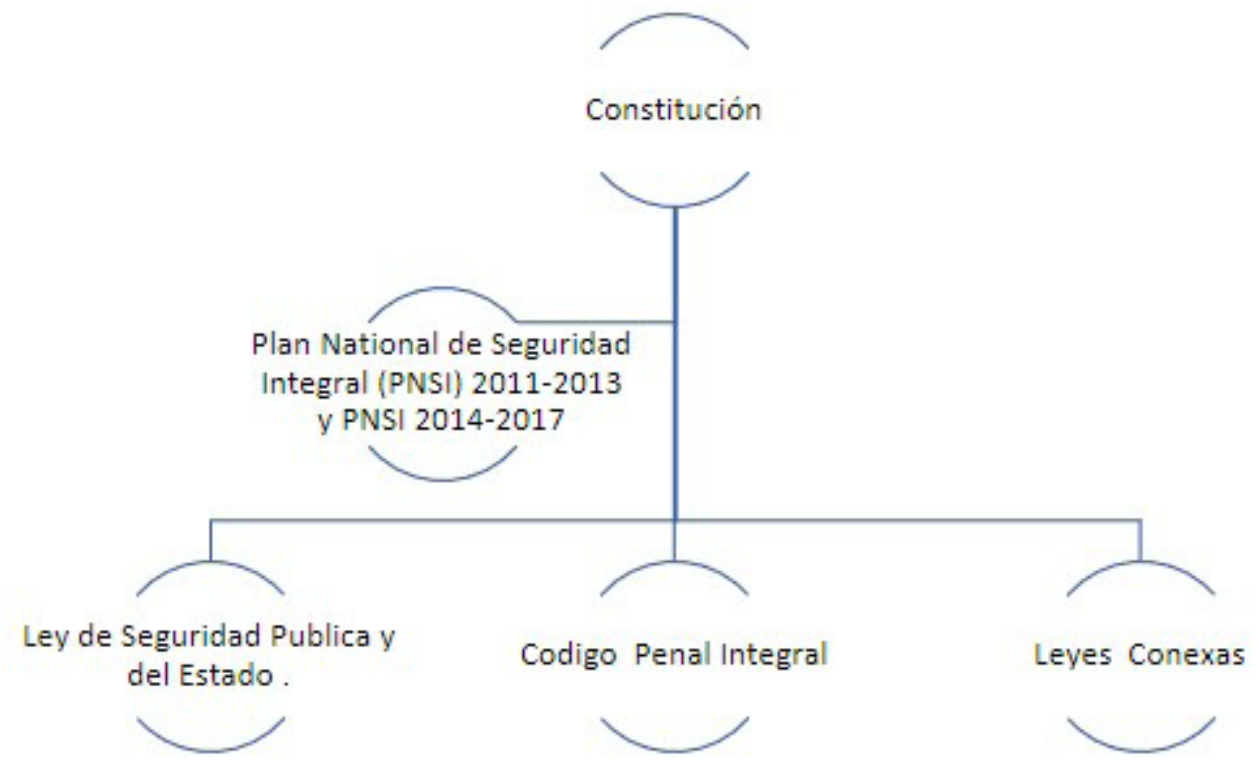

En otro orden de cosas, la gran influencia que dispuso EE.UU. en la soberanía ecuatoriana se percibió como una amenaza a la consecución de las políticas públicas del estado hacia los ciudadanos $^{47}$, ya que Ecuador se encontraba en una situación de dependencia. En este sentido, la norma normarum ha establecido un mecanismo constitucional para garantizar la seguridad sin interferencias de otras potencias. Por lo tanto, la visión a largo plazo era convertirse en un estado independiente. En este sentido, el artículo 5 de la CRE establece que: "Ecuador es un territorio de paz. No se permitirá el establecimiento de bases militares ni instalaciones extranjeras para fines militares. Está prohibido asignar bases militares nacionales a fuerzas armadas o de seguridad extranjeras”. Este artículo se basó en la premisa de que EE.UU., a través de su Comando Sur, disponía de medios tecnológicos para realizar interferencias $y$, por tanto, realizaba espionaje en la base militar en Manta —una ciudad portuaria ubicada en la costa del Pacífico de Ecuador-, que servía como centro para actividades militares, información sobre rutas y lucha contra el narcotráfico.

Sin embargo, una vez que se aprobó la constitución, se retiró la base estadounidense y retomó el control la Fuerza Aérea Ecuatoriana, además de que se evitó cualquier forma de cooperación y entrenamiento militar ${ }^{48}$. En el mismo sentido, en términos de inteligencia, las unidades del ejército y la policía pasan por el control de un órgano civil dirigido por el Ejecutivo,

\footnotetext{
47 lbíd., art. 416.

48 La declaración de persona non grata del embajador de los Estados Unidos fue el punto fuerte de la tensión entre Quito y Washington, que derivaba de la información de espionaje por parte de la embajada estadounidense según Wikileaks.
} 
por lo que la creación de la Secretaría de Inteligencia se colocó bajo los auspicios de la Presidencia de la República ${ }^{49}$.

Esta estrategia de implementación de un marco soberano se tradujo por un viraje de 360 grados en relación a los aliados estratégicos en temas de seguridad.Así pues, países como EE.UU., Francia, Inglaterra e Israel, fueron reemplazados por países como Rusia, China, Irán y Venezuela. De esta manera se busco establecer un enfoque de cooperación Sur-Sur en la constitución, por lo que la norma proclama la independencia y la igualdad jurídica de los estados, la coexistencia pacífica y la autodeterminación de los pueblos; así como cooperación, integración y solidaridad ${ }^{50}$.

Además del discurso del Ejecutivo en torno a la política de seguridad, se crearon nuevos paradigmas de cooperación para luchar contra las amenazas, de modo que la integración regional fue un mecanismo para coordinar acciones conjuntas en la relación Sur-Sur. La agenda de defensa y seguridad regional de América del Sur según Flemes, Nolte y Wehner, comenzó con la creación de UNASUR, un proceso de integración regional que buscó en sus primeros días crear una comunidad de seguridad regional. Desde la creación del Consejo de Defensa liderado por la dupla Brasil-Argentina, su objetivo principal fue combatir las amenazas como el terrorismo, la corrupción, el problema mundial de las drogas, el tráfico de personas, el tráfico de armas pequeñas y ligeras, la delincuencia organizada transnacional y otras amenazas, así como el desarme, la no proliferación de armas nucleares y la destrucción masiva y la remoción de minas ${ }^{51}$. Por otro lado, este proceso de integración buscó producir sentimientos compartidos de seguridad a partir del intercambio de información y experiencias en el campo de la defensa ${ }^{52}$. Sin embargo, en la actualidad, este proceso de integración ha sufrido un gran revés puesto que varios de sus miembros han denunciado el tratado constitutivo debido al viraje ideologico hacia la derecha.

Tabla III.Tipos de seguridad y definiciones en la Constitución del Ecuador.

\begin{tabular}{|l|l|l|}
\hline Tipos de seguridad & Definición & Discurso- norma \\
\hline Seguridad Integral & $\begin{array}{l}\text { Condición que tiene como objetivo garantizar y pro- } \\
\text { teger los derechos humanos y las libertades de los } \\
\text { ecuatorianos, la gobernabilidad, la aplicación de la } \\
\text { justicia, el ejercicio de la democracia, la solidaridad, la } \\
\text { reducción de vulnerabilidades, la prevención, la pro- } \\
\text { tección, intervención y remediación ante riesgos y y } \\
\text { amenazas. }\end{array}$ & $\begin{array}{l}\text { Artan nacional de Seguri- } \\
\text { dantegral. }\end{array}$ \\
\hline Seguridad Humana & $\begin{array}{l}\text { Asegurar la convivencia pacífica de las personas, la } \\
\text { promoción de una cultura de paz y la prevención de } \\
\text { formas de violencia y discriminación y la comisión de } \\
\text { delitos y delitos. CRE }\end{array}$ & $\begin{array}{l}\text { Seguridad Humana: Título VII, } \\
\text { Unen del Buen Vivir, Capítulo } \\
\text { del LSPE } 340 \text { y 393. Preámbulo }\end{array}$ \\
\hline
\end{tabular}

\footnotetext{
49 LSPE art. 13.

${ }^{50}$ CRE, art. 4I6, I.

51 WEHNER, Leslie, FLEMES, Daniel, y Detlef NOLTE, "Una Comunidad de Seguridad Regional en formación: La UNASUR y su Consejo de Defensa" en Estudios Internacionales, vol. 44, n I70, 201 I, pp. I05-I 27.

52 Véase Tratado de UNASUR.
} 


\begin{tabular}{|l|l|l|}
\hline Seguridad Pública y del & $\begin{array}{l}\text { Tiene como objetivo promover una sociedad que lo- } \\
\text { gre el bienestar, el bienestar y el desarrollo integral, } \\
\text { con un Estado que asuma sus responsabilidades y } \\
\text { una sociedad activa que contribuya a estos objetivos, } \\
\text { para lo cual es necesario implementar diferentes ti- } \\
\text { pos de seguridad en la seguridad. público }\end{array}$ & Preámbulo le laP \\
\hline Seguridad ciudadana, & $\begin{array}{l}\text { Política estatal dirigida a fortalecer y } \\
\text { modernizar los mecanismos necesarios para garanti- } \\
\text { zar los derechos humanos, en particular el derecho a } \\
\text { vida sin violencia y crimen, reduciendo los niveles de } \\
\text { crimen, protegiendo a las víctimas } \\
\text { y mejorar la calidad de vida de todos los habitantes } \\
\text { del Ecuador. }\end{array}$ & Art 23 LSPE \\
\hline Seguridad Nacional & $\begin{array}{l}\text { Orientada hacia la supervivencia del estado, la defen- } \\
\text { sa de su soberanía, integridad territorial e intereses } \\
\text { nacionales (expresados en términos de poder ofen- } \\
\text { sivo y disuasorio) }\end{array}$ & $\begin{array}{l}\text { Plan de Seguridad } \\
\text { nacional 20I4-20I7 }\end{array}$ \\
\hline
\end{tabular}

En este sentido, el viejo concepto de seguridad nacional tenía como único propósito la supervivencia de la ideología capitalista a través del estado y de las élites nacionales. Este era un término de status quo criticado por los proponentes de un concepto más amplio llamado seguridad integral, que apuntaba a la protección y materialización de los derechos y libertades fundamentales, el cual fue concebido en los debates de organismos internacionales sobre seguridad. En la constitución actual, si bien no se hace mención del concepto de seguridad nacional, su esencia se transpuso en el concepto de seguridad pública y del estado que, tan solo en una parte del concepto general de seguridad integral, hace alusión a una visión antropocéntrica.

\section{Conclusión}

En base a la teoría estructuralista, las ideologías creadas en el centro-mundo disponen de un flujo y absorción directa en la periferia debido a la capacidad de influencia que disponen las potencias. Es así como, en este caso de estudio, tanto la doctrina de seguridad nacional establecida por EE.UU., así como la Seguridad Integral o denominada Humana — vehiculada por Naciones Unidas y potencias medias-, han sido implantadas de manera directa en la normativa jurídica ecuatoriana por militares, académicos y elites políticas.

Las RRII de confrontación bipolar desarrolladas en la Guerra Fría determinaron la creación y materialización de la doctrina de seguridad nacional y, por tanto, la Republica del Ecuador al ser un aliado de EE.UU. transpuso esta doctrina en la esfera normativa nacional y en la política pública de contención y contrainsurgencia desde los años 60 hasta el 2008. Así mismo, una visión soberanista de la constitución actual introdujo una visión más global de las amenazas y planteó una respuesta en la materialización de oportunidades para el hombre en sus diversas perspectivas.

La evolución del concepto se concretiza desde una fórmula que contenía elementos ligados a la supervivencia del estado y su soberanía a través de la consecución de poder, con el fin de llegar a imponer sus intereses propios en un mundo anárquico — hacia una visión posterior a 
la caída del Muro de Berlín donde los organismos internacionales adoptan una visión más amplia de la seguridad interrelacionada de manera antropocéntrica-. De esta manera, la evolución del concepto de seguridad nacional ha atravesado un proceso de confrontación hacia un mecanismo de cooperación entre estados.

Bibliografía

BALZACQ,Thierry, “Qu'est-ce que la sécurité nationale?” en Revue internationale et stratégique, vol. 52, n 4, 2003, pP. 33-50.

BRAUCH, Hans Günter, Threats, Challenges, Vulnerabilities and Risks. En Environmental and Human security, United Nations University: Institute for Environment and Human Security, 2005.

BRAUDEL, Fernand, La Dynamique du capitalisme, Les Éditions Artaud, París, 1985.

BURBANO DE LARA, Fernando "Todo por la patria. Refundación y retorno del estado en las revoluciones bolivarianas" en Íconos. Revista de Ciencias Sociales, $n^{\circ}$ 52, 20I5, pp. 19-4I.

BUZAN, Barry, People, States and Fear:An Agenda for International Security Studies in the Post-ColdWar Era, Lynne Rienner Publishers, Boulder, 1991.

CARR, Edward H., The Twenty Years' Crisis, 1919-1939: An Introduction to the Study of International Relations, Macmillan, Londres, 1940.

CASTAÑEDA, Jorge, "Latin America and the End of the Cold War" en CrossCurrents, vol. 4I, n 2, I99I, pp. $195-219$.

CONSTITUCIÓN DE LA REPUBLICA DEL ECUADOR, Registro Oficial 449 de 20-oct-2008.

GALLIE, Walter B., "Essentially Contested Concepts" en Proceedings of the Aristotelian Society, vol. 56, $\mathrm{n}^{\circ}$ I, I955, pp. 167-198.

HERNÁNDEZ, Rafael, “Commentary: Alliances and Dis-Alliances between the United States and Latin America and the Caribbean" en Latin American Perspectives, vol. 38, n 4, 20 I I, pp. I 3 I-I 36.

HOBBES, Thomas, y GASKIN, John C.A., Leviathan, Oxford University Press, Oxford, 1998.

HURRELL, Andrew, "Security in Latin America" en International Affairs, vol. 74, n 3, 1998, pp. 529-546.

JABLONSKY, D., et.al., Army War College, Clarke Center for the Interdisciplinary Study of Contemporary Issues, 1997.

KHATCHADOURIAN, Haig, “Vagueness” en The Philosophical Quarterly, vol. I2, n² 2, I962, pp. I38-I52.

LIPPMANN, Walter, U.S. Foreign Policy: Shield of the Republic, Little, Brown and Co., Boston, 1954.

MCMAHON, Robert, "US national security policy from Eisenhower to Kennedy” en LEFFLER, Melvin P. y WESTAD, Odd Arne (eds.), The Cambridge History of the Cold War, Cambridge University Press, Cambridge, 20I I.

MORGENTHAU, Hans J., THOMPSON, Kenneth W., y CLINTON, David, Politics Among Nations:The Struggle for Power and Peace, McGraw-Hill Higher Education, Boston, 2006

PHAM, J. Peter, "What Is in the National Interest? Hans Morgenthau's Realist Vision and American Foreign Policy” en American Foreign Policy Interests, vol. 37, $n^{\circ}$ 4, 2015, pp. I87-193.

RUBIN, F. "The Theory and Concept of National Security in the Warsaw Pact Countries" en International Affairs, vol. $58, n^{\circ} 4,1982$, pp. 648-657.

SECREATARIA DEL CONSEJO DE SEGURIDAD NACIONAL, Ley de Seguridad, 1979.

SHINODA, Hideaki, "The concept of human security: historical and theoretical implications" en Conflict and human security: A search for new approaches of peace-building, IPSHU English Research Report Series, $n^{\circ}$ 9, 2004, PP. $5-22$.

SINGER, J. David, “Threat-perception and the Armament-Tension Dilemma” en The Journal of Conflict Resolution, vol. 2, $\mathrm{n}^{\circ}$ I, 1958, pp. 90-105.

STONE, Marianne, "Security According to Buzan:A Comprehensive Security Analysis" en Spring, n 5, 2009.

TRAGER, Frank N., y KRONENBERG, Philip S., National Security and American Society;Theory, Process, and Policy, National Security Education Program, University Press of Kansas, Kansas City, 1973.

TULCHIN, Joseph, “Los Estados Unidos y América Latina en la década del 60” en Estudios Internacionales, vol. 21 , $\mathrm{n}^{\circ}$ 84, I 988, pp. 462-497.

VAISSE, Maurice, Les relations internationales depuis 1945, Armand Colin, París, 1996.

WALT, Stephen M., The Renaissance of Security Studies, Sage, Beverly Hills, I99I.

WALTZ, Kenneth N., Man, the State and War:A Theoretical Analysis, Columbia University Press, Nueva York, 1959.

WEHNER, Leslie, FLEMES, Daniel,y Detlef NOLTE,“Una Comunidad de Seguridad Regional en formación: La UNASUR y su Consejo de Defensa” en Estudios Internacionales, vol. 44, n I 70, 20 I I, pp. I 05-I 27.

WOLFERS, Arnold, Discord and Collaboration: Essays on International Politics, Johns Hopkins Press, Baltimore, 1968.

WOLFERS, Arnold, National Security As an Ambiguous Symbol, National Emergency Training Center, Emmitsburg, 1952. 


\section{RELACIONES INTERNACIONALES}

Revista académica cuatrimestral de publicación electrónica Grupo de Estudios de Relaciones Internacionales (GERI)

Universidad Autónoma de Madrid, España

https://revistas.uam.es/relacionesinternacionales

ISSN 1699 - 3950

f facebook.com/RelacionesInternacionales

3. twitter.com/RRInternacional

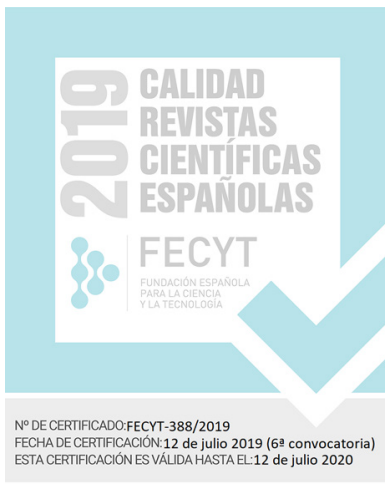

